\title{
Porous Graphitized Carbon for Adsorptive Removal of Benzene and the Electrothermal Regeneration
}

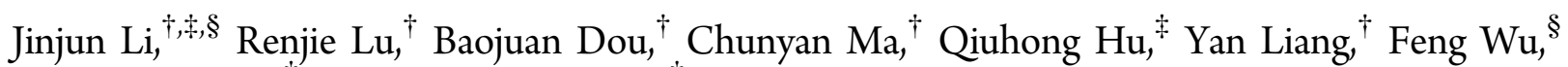 \\ Shizhang Qiao, ${ }^{*, *}$ and Zhengping Hao ${ }^{*} \dagger$ \\ ${ }^{\dagger}$ Research Center for Eco-Environmental Sciences, Chinese Academy of Sciences, Beijing 100085, China \\ ${ }^{\ddagger}$ ARC Centre of Excellence for Functional Nanomaterials, Australian Institute for Bioengineering and Nanotechnology, The \\ University of Queensland, QLD 4072, Australia \\ ${ }^{\S}$ School of Resource and Environmental Science, Hubei Key Laboratory of Biomass-Resources, Wuhan University, Wuhan 430079, \\ China
}

Supporting Information

ABSTRACT: Graphitized carbons with mesoporous and macroporous structures were synthesized by a facile template-catalysis procedure using resorcinol and formaldehyde as carbon precursors and particulate hydrated metal oxides as both template and catalyst precursors. The materials were used as novel adsorbents for low-concentration benzene vapor. Furthermore, on the basis of the good electrical conductivities associated with the graphitized structures, an electrothermal desorption technique, which involved passing electric currents through the adsorbents to generate Joule heat, was employed to regenerate the saturated adsorbents and produce enriched benzene vapors. In comparison to microporous activated carbon, the porous graphitized carbons could
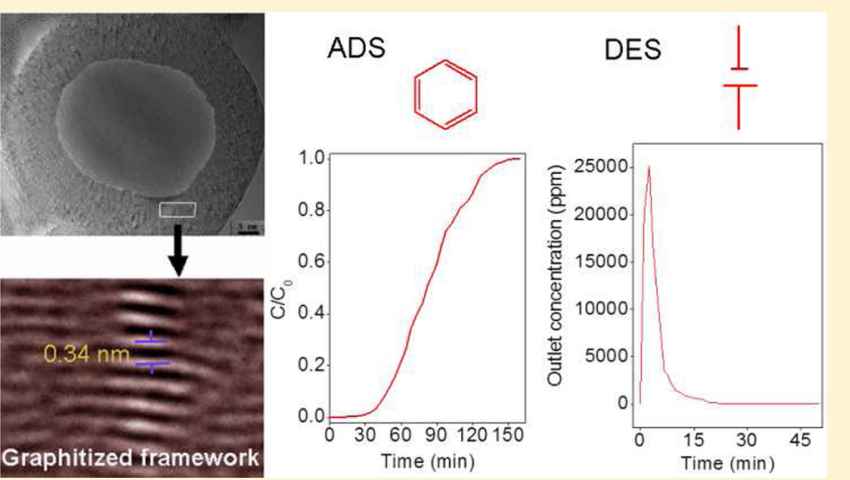

afford a much quicker and more efficient regeneration by electrothermal desorption technique due to their enhanced conductivity and larger pore sizes. In addition, the concentration of the desorbed organics could be controlled by adjusting the applied voltages, which might be interesting for practical secondary treatment. It is promising that the joint utilization of porous graphitized carbon adsorbents and electrothermal desorption technique might develop effective and energy-saving processes for VOCs removal.

\section{INTRODUCTION}

Volatile organic compounds (VOCs) from industrial emissions are one of the most troublesome air pollutants due to their toxicity and malodor, as well as their precursor roles in photochemical formation of ozone and smog in the boundary layer. ${ }^{1}$ In many cases, the difficulties in disposing of VOCs emissions lie in the low concentration of organic vapors, which hinder their condense recovery or destructive combustion. Therefore, the lean organic vapors are often required to be enriched by adsorption before they are subjected to secondary treatment. At present activated carbons are widely used as adsorbents due to their high adsorption capacity. The commercial activated carbons are generally dominated by microporous structures, and their pore sizes are often close to the molecule sizes of VOCs, and therefore their pore channels can strongly hold VOCs molecules because all the surrounding pore wall surfaces can induce van der Waals forces with the captured molecules. However, the extremely strong adsorption affinities often cause difficulties in the desorption of VOCs by a general hot-air stripping process.
Researchers have tried to search for alternative adsorbents which can afford not only effective adsorption but also quick desorption of VOCs. ${ }^{2-6}$ Mesoporous silicate-based materials with high surface areas were considered to be good candidates because they have significantly larger pore diameters than activated carbons. $^{2-6}$ However, under humid conditions their hydrophilic surfaces with abundant silanol groups lead to great loss in adsorptive capacity because of the preferential adsorption toward water. ${ }^{2}$ Although some attempts have been made to modify mesoporous silica surfaces with hydrophobic functional groups or to synthesize mesoporous inorganic/ organic hybrid materials, the synthesis of such materials seems still quite complex and high in cost. ${ }^{3-6}$

On the other hand, some environmental engineers have tried to find other effective desorption technologies. On the basis of the electrical conductivity of activated carbons, some pioneer-

Received: July 27, 2012

Revised: September 28, 2012

Accepted: October 23, 2012

Published: October 23, 2012 
ing researchers proposed an electrothermal desorption technique (EDT), ${ }^{7-12}$ namely, passing electric current directly through the activated carbons to generate Joule heat and induce desorption of adsorbed VOCs molecules. In comparison with traditional desorption methods, the EDT was considered to possess unique advantages including high energy efficiency, rapid heating rate and mass transfer kinetics, maximized concentration of desorbed adsorbate, and simple and compact equipment. ${ }^{7-12}$ Nevertheless, actually little progress has been made for the development of EDT, and one major reason could be that the electrical conductivities of the activated carbons are actually very limited. Up to now other adsorbents have never been utilized with EDT because they are hardly electrically conductive.

Herein, we propose that porous graphitized carbons (PGCs) could be used as alternative adsorbents for VOCs. It is known that perfect graphite materials have crystalline structures with long-range hexagonal stacking and they are generally almost nonporous. In contrast, activated carbons contain abundant micropores with some micrograins of crystalline graphite in their frameworks, and they only show short-range ordering in these micrograins. Differently, the porous graphitized carbons appearing recently contain lots of mesopores whose frameworks show long-range ordering that is similar to graphite although many of them appear somewhat turbostratic. ${ }^{13-18}$ The graphitized structures ensure good hydrophobicity ${ }^{19}$ and thermal stability, ${ }^{20}$ which is desirable in adsorptive removal of VOCs. Furthermore, the PGCs have improved electrical conductivities due to their well-crystallized structures, ${ }^{21}$ therefore they might be more suitable for the utilization of EDT.

However, the synthesis of PGCs remains a great challenge at present. The thermal graphitization of amorphous carbon needs a high temperature of more than $2200{ }^{\circ} \mathrm{C},{ }^{22,23}$ which is likely to induce nonporous crystallized materials. Recently, some researchers found that porous carbons with fairly graphitic structure could be obtained at temperatures lower than 1300 ${ }^{\circ} \mathrm{C}$ by utilizing porous silicas as hard-templates and special carbon precursors like fused aromatics as carbon precursors, $^{13-18}$ however it seemed difficult to achieve large-scale production because such hard-template strategies have some disadvantages including very complicated processes, high cost of sacrificial hard template, and great difficulty in removing template. Inagaki et al. ${ }^{24,25}$ used magnesium oxide particles as templates to synthesize porous materials, and the metal oxide templates were easily removed by acid, whereas the products were generally amorphous. Recently, Shen et al. ${ }^{26}$ reported that in the presence of iron salts, magnesium oxide could template the formation of porous carbon with graphitized structure at $1000{ }^{\circ} \mathrm{C}$ because the iron was graphitization catalyst, whereas it seemed that the template particles posed a negative effect on the graphitization degree.

In this paper, we present a new way to synthesize PGCs using some easily prepared hydrolyzed metal species as both pore templates and graphitization catalysts with some common chemicals (resorcinol and formaldehyde) as carbon precursors. The prepared PGCs were used as adsorbent for benzene, a model pollutant of VOCs. Moreover, for the first time, EDT was performed on PGCs for VOCs enrichment and removal.

\section{EXPERIMENTAL SECTION}

Chemicals. Resorcinol (99.5\%), cobalt nitrate (99.0\%), and iron nitrate (98.5\%) were purchased from Shantou Xilong Chemical Company. Hydrochloric acid (36.0-38.0\%), man- ganese nitrate (49.0-51.0\% aqueous solution), sodium hydroxide (96.0\%), and formaldehyde (37.0-40.0\% aqueous solution) were purchased from Beijing Chemical Reagents Company. Activated carbon was kindly supplied by Chemical Defense Institute of China.

Synthesis of Materials. Hydrated metal oxide nanoparticles were prepared using a method proposed recently by us with minor modifications. ${ }^{27}$ To synthesize hydrated iron oxide nanoparticles, iron nitrate $(0.04 \mathrm{~mol})$ and sodium hydroxide $(0.16 \mathrm{~mol})$ aqueous solutions were mixed together to produce iron hydroxide. After centrifugation, glacial acetic acid was added to the precipitated hydroxide slurries (the molar ratio of acetic acid to iron was 3). Afterward the mixture was continuously stirred for more than $1 \mathrm{~h}$ until a stable aqueous solution containing nanosized hydrated iron oxide species (about $30 \mathrm{~mL}$ ) formed. Resorcinol (2.3 g) and formaldehyde (5 g, 37.0-40.0\%) were then added to the above solution, and the mixture was aged statically at $50{ }^{\circ} \mathrm{C}$ to produce a composite of resin polymer and iron oxide particles. The solid material was obtained via centrifugation and then dried in an oven at $100{ }^{\circ} \mathrm{C}$. The dried composite was carbonized at $900{ }^{\circ} \mathrm{C}$ for $2 \mathrm{~h}$ under a nitrogen flow $(30 \mathrm{~mL} / \mathrm{min})$, then it was soaked in concentrated hydrochloric acid (36.0-38.0\%) overnight to remove iron template, forming final product labeled as CFe. By similar procedures, CCo and CMn were also prepared.

Characterization Techniques. X-ray diffraction (XRD) patterns were recorded on Siemens D5005 diffractometer using $\mathrm{Cu}-\mathrm{K} \alpha$ radiation. Raman spectra were collected on Renishaw RM2000 microscopic confocal Raman spectrometer using a $\mathrm{He}-\mathrm{Ne}$ laser source $(\lambda=632.8 \mathrm{~nm})$. Transmission electron microscopy (TEM) images were taken on a Tecnai F20 at an acceleration voltage of $200 \mathrm{kV}$. Nitrogen sorption isotherms at liquid nitrogen temperature were obtained on NOVA1200 gas sorption analyzer. The BET surface area was calculated using experimental points at a relative pressure of $P / P_{0}=0.05-0.25$. Total pore volume was obtained by the nitrogen amount adsorbed at $P / P_{0}=0.99$.

Cyclic Dynamic Adsorption/Electrothermal Desorption of Benzene. The cyclic adsorption/electrothermal desorption of benzene on porous graphitized carbons was conducted in a continuous flow tube reactor made of iron with inner diameter of $9 \mathrm{~mm}$ (see Supporting Information). At the center of this reactor there was an iron rod with diameter of 3 $\mathrm{mm}$, which was coaxial to the iron tube. The iron tube and iron rod were used as electrodes connected to a DC power source. The tube and the rod were kept separated by silicon rubber rings at both ends. In the adsorption experiment, carbon adsorbent $(250 \mathrm{mg}$, pelletized to $40-60$ mesh) was packed in the space between the tube and the rod, and a $140 \mathrm{~mL} \mathrm{~min}^{-1}$ nitrogen flow containing $800 \mathrm{ppm}$ benzene vapor was then passed through the reactor until the adsorption equilibrium was reached. In the desorption experiment, a voltage was put on the adsorbents and a nitrogen flow $\left(30 \mathrm{~mL} \mathrm{~min}{ }^{-1}\right)$ was used as carrier gas, and the outlet temperature was monitored by a resistance thermometer sensor. The benzene concentrations in the outlet gases in both adsorption and desorption experiments were online-monitored by a gas chromatograph equipped with a hydrogen flame ionization detector (FID) and a six-way injection valve, and an empty separation column was employed in order to achieve a quick analysis. During the desorption run, a special self-made smaller loop was used for sampling in order to fit the high concentration of the desorbed benzene. The 
adsorption/desorption experiments were repeatedly performed several times.

\section{RESULTS AND DISCUSSION}

In this work, we used resorcinol and formaldehyde as carbon precursors because they can polymerize in aqueous solution to form resin network, which can convert to carbon materials after carbonization at relatively high temperatures. In a previous work, ${ }^{27}$ we have shown that the hydrated metal oxide nanoparticles are several nanometers in size as revealed by TEM images (see Supporting Information). The prepared hydrated metal oxide nanoparticles have hydroxyl groups on their surfaces, which can induce hydrogen-bond interaction with the dissolved resorcinol and formaldehyde. Therefore, during the polymerization process, these nanoparticles can be easily incorporated into the formed polymer network, forming a polymer hybrid containing dispersed metal nanoparticles. During carbonization at $900{ }^{\circ} \mathrm{C}$ in nitrogen, the metal oxides are carbothermally reduced to metals, which might be capable of catalyzing the graphitization of the carbonaceous framework. The final acid digesting could remove the embedded metal particles, leaving porosities in the products.

Figure 1 shows the X-ray diffraction (XRD) patterns of the prepared materials $\mathrm{CFe}, \mathrm{CCo}$, and $\mathrm{CMn}$. The well-defined

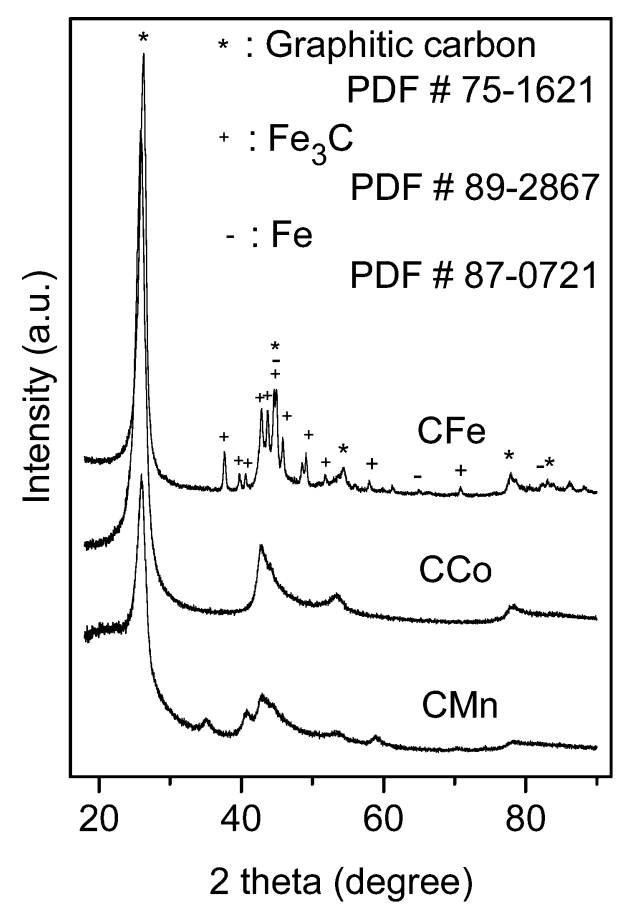

Figure 1. X-ray diffraction patterns of the carbon materials.

diffraction peaks at $26,44,54$, and $78^{\circ}$ can be respectively indexed as the (002), (10), (004), (110) diffraction for typical graphitic materials, ${ }^{14,15}$ suggesting that these materials have graphitized structures. The interlayer spacing values $\left(d_{002}\right)$ is an important parameter to evaluate the quality of the graphitization. The $d_{002}$ values derived from the XRD profiles are shown in Table 1, and it is known that the perfect hexagonal graphite has a $d_{002}$ value of $3.354 \AA^{28}$ Apparently CFe shows the best graphitized structure among the three samples because its $d_{002}$ value is the closest to that of perfect hexagonal graphite. The slight deviation of $d_{002}$ values from perfect graphite indicates that these porous materials have turbostratic characteristics. In
Table 1. Physical and Textural Properties of the Porous Carbons

$\begin{array}{lcccc}\text { sample } & d_{002}(\AA) & S_{\mathrm{BET}}{ }^{a}\left(\mathrm{~m}^{2} \mathrm{~g}^{-1}\right) & V_{\mathrm{p}}^{b}\left(\mathrm{~cm}^{3} \mathrm{~g}^{-1}\right) & I_{\mathrm{G}} / I_{\mathrm{D}} \\ \mathrm{CFe} & 3.377 & 361 & 0.61 & 1.12 \\ \mathrm{CCo} & 3.444 & 252 & 0.21 & 0.85 \\ \mathrm{CMn} & 3.423 & 441 & 0.50 & 0.79 \\ \mathrm{AC}^{c} & & 1114 & 0.63 & \end{array}$

${ }^{a}$ BET specific surface area. ${ }^{b}$ Total pore volume. ${ }^{c}$ Activated carbon.

the XRD patterns, the diffraction peaks associated with metal species, such as iron and iron carbide, can also be found, although the samples have been thoroughly treated by concentrated hydrochloric acid. This could be attributed to the complete covering of carbon shells on some of the metal particles, which prevent them from dissolving by acids. In addition, the materials $\mathrm{CCe}, \mathrm{CCr}, \mathrm{CZr}, \mathrm{CTi}$, and $\mathrm{CAl}$ were also prepared by similar method, however their XRD patterns indicate that these metal nanoparticles have no catalytic activity for the graphitization process under the experimental conditions (see Supporting Information).

Figure 2 shows the Raman spectroscopy of $\mathrm{CFe}, \mathrm{CCo}$, and $\mathrm{CMn}$. The vibration at $1580 \mathrm{~cm}^{-1}$ (G-band) is attributed to the

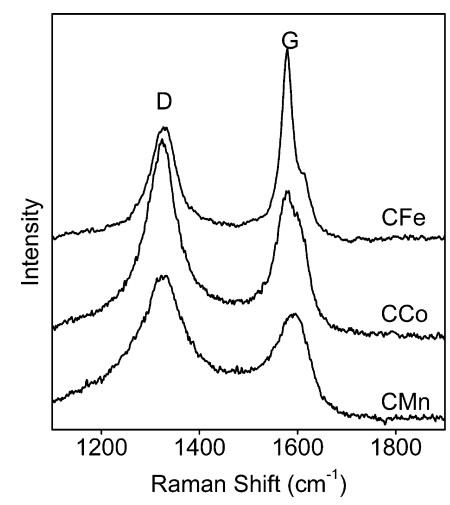

Figure 2. Raman spectroscopy of the carbon materials.

interplane $\mathrm{sp}^{2} \mathrm{C}-\mathrm{C}$ stretching in graphitized carbon, while the other one at $1327 \mathrm{~cm}^{-1}$ (D-band) is attributed to amorphous carbon. ${ }^{13}$ The calculated intensity ratios of $G$ band to $D$ band $\left(I_{\mathrm{G}} / I_{\mathrm{D}}\right)$ are listed in Table 1 . Apparently the Raman spectroscopy also indicates that $\mathrm{CFe}$ has the best graphitized structure because it shows the most intensive G-band vibration as reveled by the intensity ratios $I_{\mathrm{G}} / I_{\mathrm{D}}$.

Figure 3 shows the transmission electron microscopy (TEM) images of $\mathrm{CFe}$. They reveal the presence of cage-like mesopores

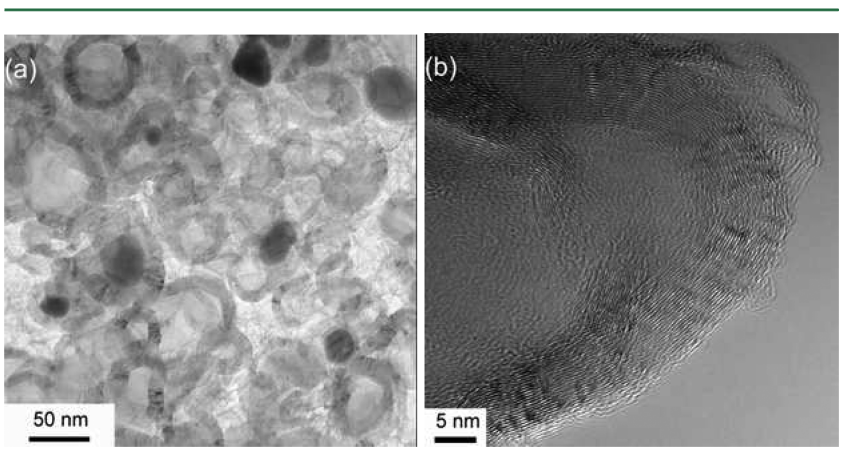

Figure 3. Transmission electron microscopy images of CFe material. 

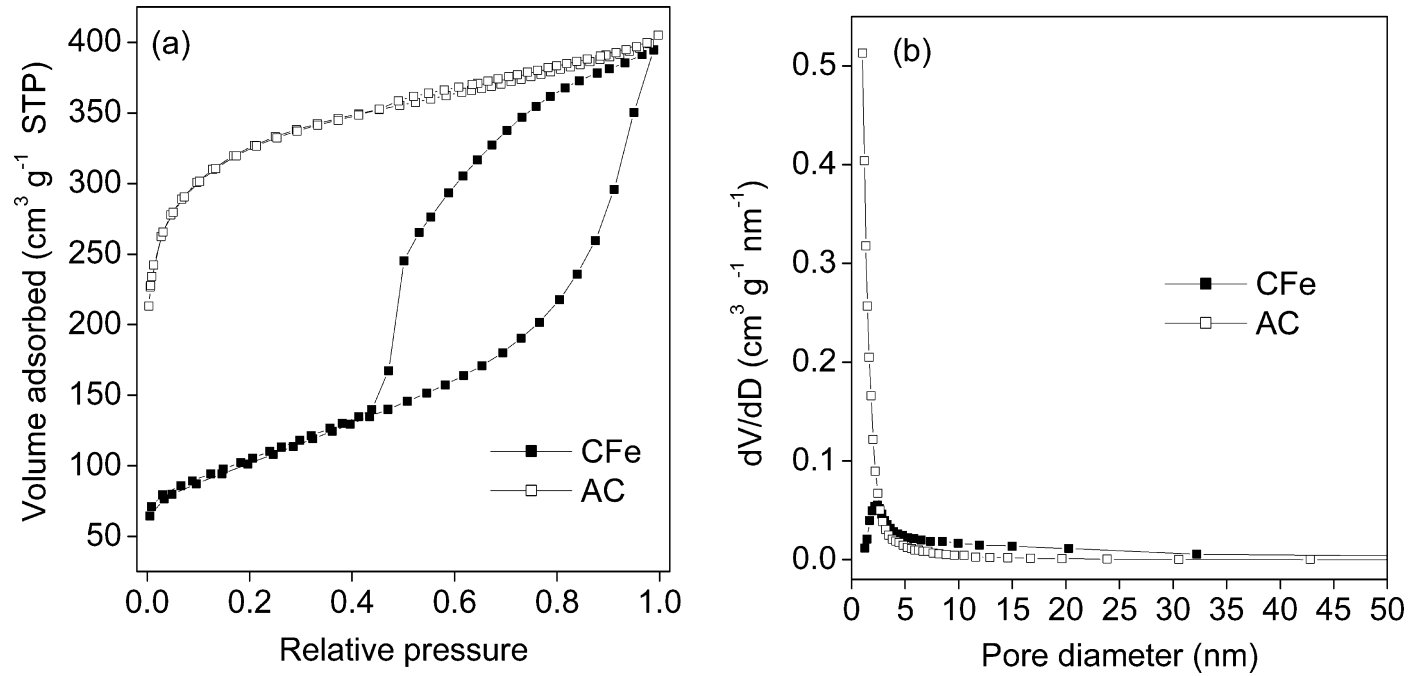

Figure 4. (a) Nitrogen adsorption-desorption isotherms and (b) BJH pore size distribution curves of CFe and activated carbon.
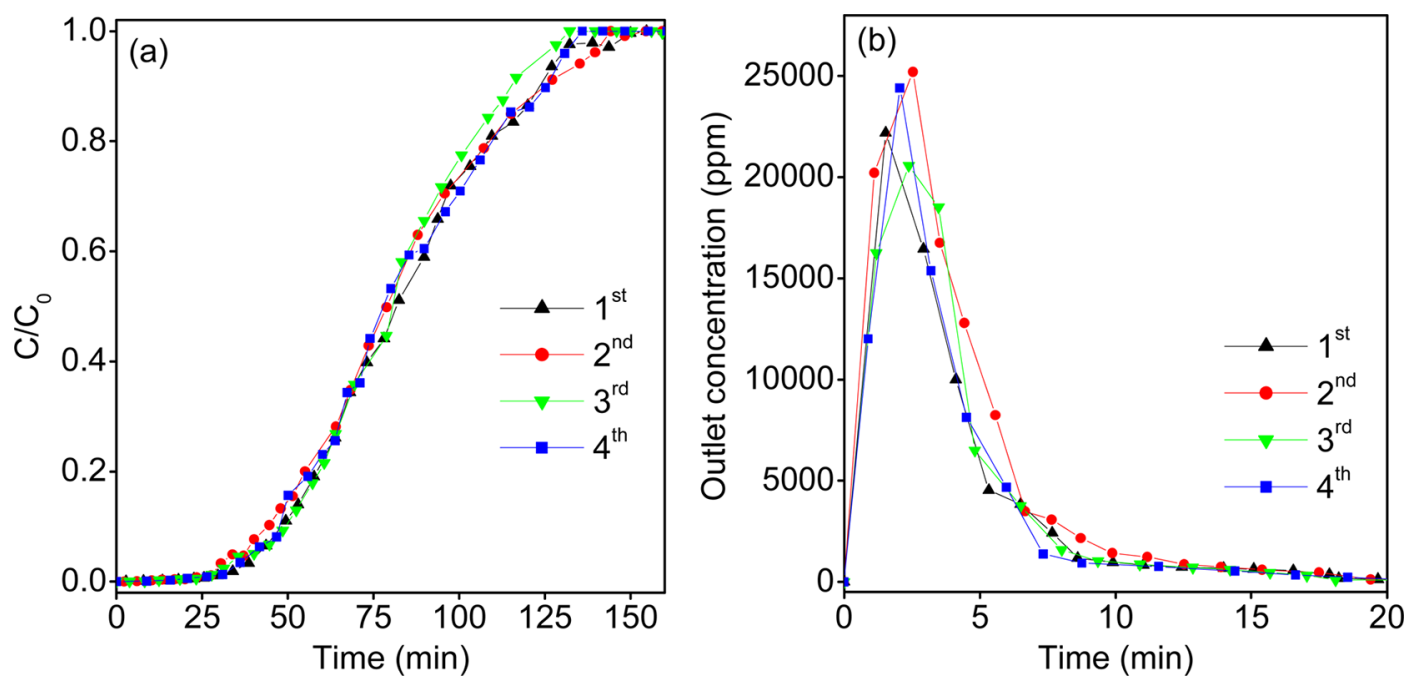

Figure 5. Cyclic adsorption/desorption profiles of benzene on CFe: (a) the breakthrough curves obtained at room temperature and (b) the electrothermal desorption curves obtained under a voltage of $3 \mathrm{~V}$.

and macropores with pore sizes ranging from 10 to $60 \mathrm{~nm}$ in this sample. The layered structure of graphitized framework is clearly observed in the high-magnification image (Figure $3 b$ ), which corroborates the results drawn from the XRD patterns and the Raman spectroscopy. Actually, the TEM images of the $\mathrm{CFe}$ sample before acid-digesting show lots of iron particles, whereas they do not show apparent mesoporous or macroporous structures (see Supporting Information). The presence of cage-like pores in the final product could be attributed to the template effect of the metallic nanoparticles.

The porosities of the materials were analyzed from the nitrogen sorption isotherms collected at liquid nitrogen temperature. For comparison purposes, the porosity of an activated carbon is also analyzed. Figure 4 shows the nitrogen isotherms and the $\mathrm{BJH}$ pore size distribution curves of the carbon materials. CFe shows a type IV nitrogen isotherm, which is typically characteristic for mesoporous materials, ${ }^{29}$ as indicated by its pore size distribution curve. In contrast, the activated carbon shows a nitrogen isotherm that is much closer to type I, which is typically characteristic for microporous solids having relatively small external surfaces, ${ }^{29}$ as revealed by its pore size distribution curve. The derived textural property data are listed in Table 1. Microporous activated carbon has a high specific surface area of $1114 \mathrm{~m}^{2} \mathrm{~g}^{-1}$. Porous graphitized carbon $\mathrm{CFe}$ has a surface area of $361 \mathrm{~m}^{2} \mathrm{~g}^{-1}$, which is rather high considering that the crystallization of carbonaceous materials during graphitization process tends to reduce porosities significantly.

The porous structures of PGCs might afford them to be utilized as adsorbents for the removal of pollutants, and the improved conductivity associated with their graphitized structures might favor electrothermal desorption of adsorbed pollutants. CFe was selected as adsorbent and benzene was selected as model pollutant of VOCs. For comparison purposes, an activated carbon was also used as another adsorbent. The cyclic dynamic adsorption and electrothermal desorption experiments were performed in a continuous flow reactor made of iron. The electrical resistances (ER) of the adsorbent beds were measured by a voltammetry method. The ER value of the $\mathrm{CFe}$ bed was ca. $0.5 \Omega$, while that of the activated carbon bed was ca.1.2 $\Omega$, indicating that the porous graphitized carbon was more electrically conductive. To desorb 

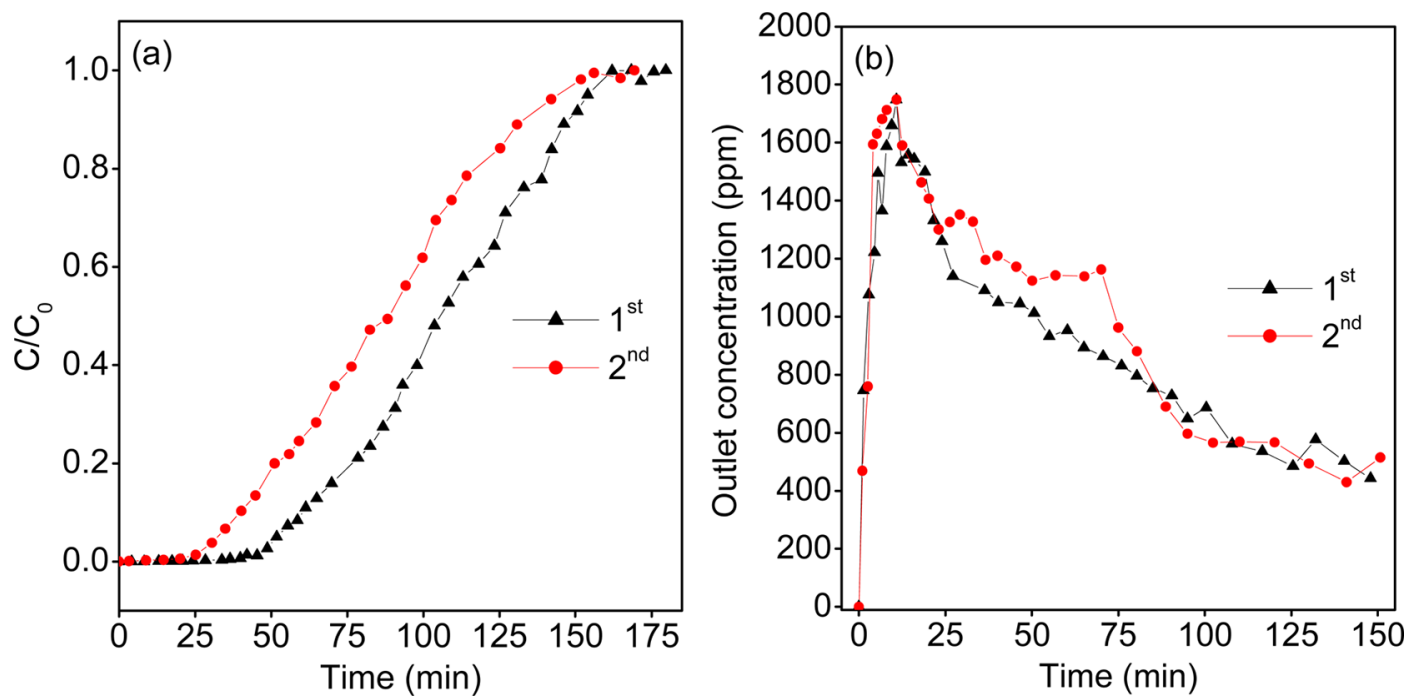

Figure 6. Cyclic adsorption/desorption profiles of benzene on activated carbon: (a) breakthrough curves obtained at room temperature and (b) electrothermal desorption curves obtained under a voltage of $4.6 \mathrm{~V}$.
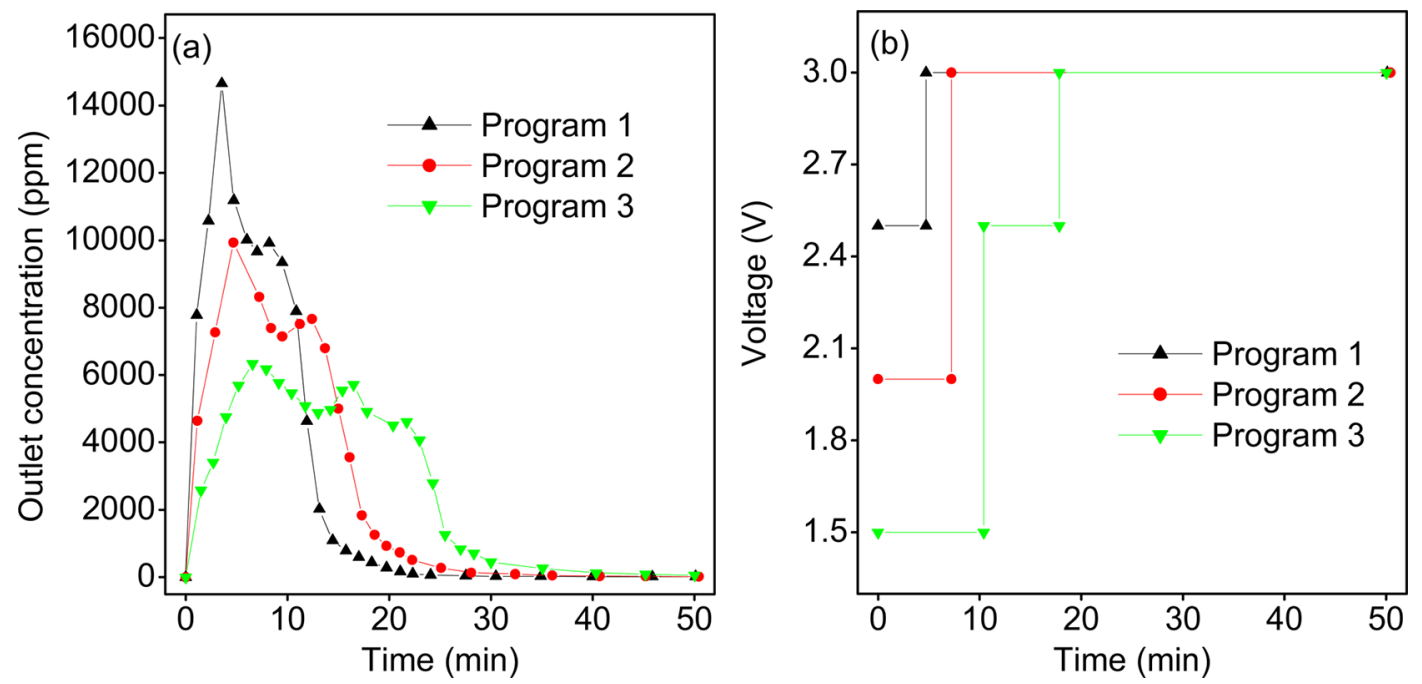

Figure 7. Desorption profiles of benzene on CFe under different voltage modes: (a) electrothermal desorption curves and (b) applied voltage programs.

the benzene adsorbed on $\mathrm{CFe}$, a voltage of $3 \mathrm{~V}$ was imposed on the adsorbents to generate Joule heat, and the calculated power was $18 \mathrm{~W}$. Whereas to desorb the benzene adsorbed on activated carbon, a voltage of $4.6 \mathrm{~V}$ was imposed to get a power that was similar to that of the CFe bed.

Figure $5 \mathrm{a}$ and $\mathrm{b}$ show the breakthrough curves for benzene adsorption on $\mathrm{CFe}$ at room temperature and the corresponded electrothermal desorption curves, respectively. Four successive adsorption/desorption runs were conducted, and the adsorption capacity calculated based on the breakthrough curves from the first run to the last run were $96.2,92.8,91.3$, and $93.6 \mathrm{mg}$ $\mathrm{g}^{-1}$, respectively. The breakthrough curves in the four runs were quite similar, suggesting that the adsorption behavior were reproduced in the repeated successive adsorption/desorption process. Whereas, the obtained desorption profiles seem somewhat different from each other in the four runs. It is worth noting that the electrothermal desorption was a very quick process under the experimental conditions, and the outlet concentration could change drastically from time to time during the first few minutes, however it was almost impossible to monitor the continuous concentration change when using gas chromatography to analyze the concentration of desorbed benzene. On the other hand, by a manual sampling it was quite difficult to catch the same time point in different desorption runs. Therefore actually the obtained desorption profiles could not perfectly reflect the real-time changes of the outlet concentration, especially in the first few minutes. Anyway, the desorption profiles reflected that most benzene was desorbed within ten minutes in each desorption run, and the instant concentration of benzene in the outlet gas could reach up to 25 $100 \mathrm{ppm}$, indicating that benzene was quickly desorbed and well concentrated. The outlet concentration dropped to below $10 \mathrm{ppm}$ within $50 \mathrm{~min}$, and the maintenance of adsorption capacity in the successive adsorption runs could suggest that the $\mathrm{CFe}$ adsorbent could be effectively regenerated by electrothermal desorption. Furthermore, the temperature monitoring indicated that the outlet temperature was generally lower than $40{ }^{\circ} \mathrm{C}$ within the first $10 \mathrm{~min}$ during the electrothermal desorption, which could be beneficial to the recovery of volatile organics by freezing condensation. 
Figure $6 \mathrm{a}$ and $\mathrm{b}$ show the breakthrough curves for benzene adsorption on activated carbon at room temperature and the corresponding electrothermal desorption curves, respectively. Two successive adsorption/desorption runs were conducted. The activated carbon has a longer breakthrough time than $\mathrm{CFe}$, indicating the former has a larger adsorption capacity. The greater adsorption capacity of the activated carbon could be associated with its larger surface areas, as shown in Table 1 . Different from $\mathrm{CFe}$, the breakthrough behavior of the activated carbon was different in successive adsorption runs. The adsorption capacity of the activated carbon was calculated to be $132.6 \mathrm{mg} \mathrm{g}^{-1}$ in the first run, and it decreased to $101.2 \mathrm{mg}$ $\mathrm{g}^{-1}$ in the second run. Although the activated carbon had a higher adsorption capacity, the desorption of benzene on it was greatly delayed. The outlet benzene concentration was much lower than in the case of $\mathrm{CFe}$, and the maximum outlet concentration was less than $1800 \mathrm{ppm}$, and even after $150 \mathrm{~min}$ the outlet concentration of benzene was still above $400 \mathrm{ppm}$. The microporous structures of the activated carbon might contribute to the retardation of benzene desorption. The benzene molecule has a relatively large diameter of about 0.6 $\mathrm{nm}$, which could be comparable to the sizes of many micropores in activated carbon, therefore the captured benzene molecules are close to the carbon wall all around them, inducing strong van der Waals forces in the small hole. In contrast, for mesoporous adsorbents, the benzene molecules only have adsorption forces with the sites where they are anchored because the pore sizes are much larger than the molecule sizes.

Figure 7 shows the desorption profiles of benzene on $\mathrm{CFe}$ under programmed voltages. Before each desorption experiment, the adsorbent bed was treated by $140 \mathrm{~mL} \mathrm{~min}^{-1}$ nitrogen flow containing $800 \mathrm{ppm}$ benzene vapor for $3 \mathrm{~h}$. Figure $5 \mathrm{~b}$ indicates that a high voltage of $3 \mathrm{~V}$ tends to quickly produce a high-concentration benzene vapor in the first few minutes, and then the concentration quickly drops. Figure 7 indicated that the maximum outlet concentration of benzene could be lowered by decreasing the initial voltage owing to the decreased power to generate Joule heat, and the desorption rate could be controlled by applying programmed voltages. Especially, via properly elevating the voltage step by step, it is possible to get a relatively stable concentration of benzene vapor; for example, program 3 in Figure 7 produced a relatively stable benzene flow with a concentration mainly within $4000-6000 \mathrm{ppm}$. This could be interesting for those secondary devices such as combustor or biofilter, which require controlled release of volatile organics because sometimes a suddenly increased high concentration of organics would cause overloading for treatment.

Figure 8 shows the successive breakthrough curves for benzene adsorption on $\mathrm{CFe}$ under a humid condition in the presence of $4700 \mathrm{ppm}$ water vapor at room temperature, and the other conditions were the same as the above dry conditions. After each adsorption run, $3 \mathrm{~V}$ was imposed on the adsorbent bed for $150 \mathrm{~min}$ to desorb the adsorbed benzene and water before the next adsorption run. The breakthrough curves of the successive three runs are similar, and the calculated adsorption capacity was $82.4,78.3$, and $79.7 \mathrm{mg} \mathrm{g}^{-1}$ for the successive three runs, respectively. This indicated that approximately $85 \%$ of the adsorption capacity was still retained under the humid condition, suggesting the porous graphitized carbon is rather water-resistant. In addition, the maintenance of the adsorption capacity in successive runs indicated that water could also be

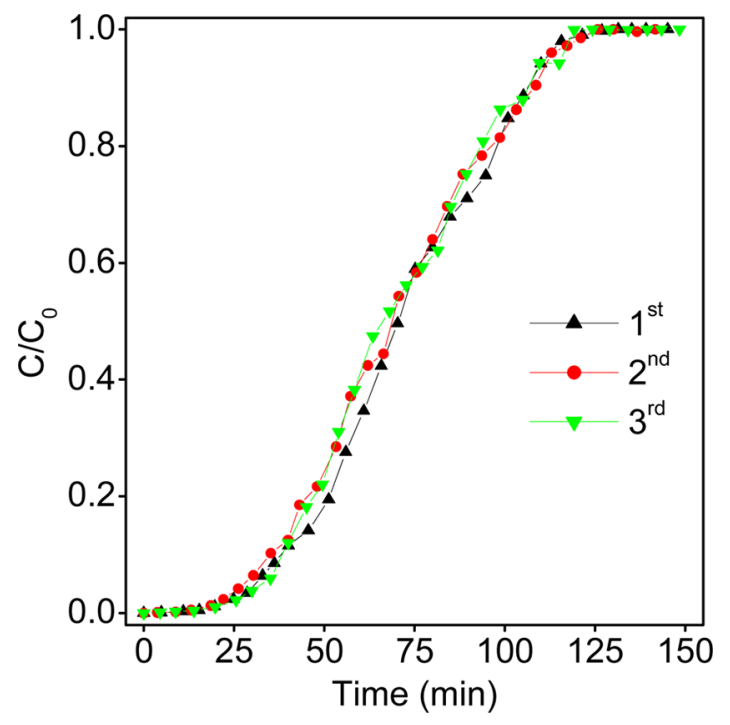

Figure 8. Successive breakthrough curves for benzene adsorption on $\mathrm{CFe}$ in the presence of $4700 \mathrm{ppm}$ water vapor at room temperature.

effectively desorbed under the employed electrothermal condition. It was reported that the graphitized structure could ensure a high hydrophobicity, ${ }^{19}$ which could be a desirable feature for those applications under humid conditions.

In this paper, the PGCs were synthesized by a simple template-catalysis method, and they could be easily produced in large scale. The improved electrical conductivities of PGCs are beneficial to EDT because they allow quick production of Joule heat. In an electrothermal desorption process, the heat is produced in the adsorbent itself and can be directly conducted to the adsorbed molecules without excess energy loss, and the outlet concentration and the temperature of adsorbent bed can be controlled by regulating the applied voltages. Therefore, EDT with the utilization of PGCs might have great potential in developing an energy-saving and efficient technique and process for VOCs disposal.

\section{ASSOCIATED CONTENT}

\section{Supporting Information}

Sketch of electrothermal desorption experiment rig (Figure $\mathrm{S} 1)$; XRD patterns of the carbon materials prepared using various hydrated metal oxide as templates (Figure S2); TEM images of the hydrated metal oxide nanoparticles (Figure S3); TEM images of CFe material before removal of the template (Figure S4). This material is available free of charge via the Internet at http://pubs.acs.org.

\section{AUTHOR INFORMATION}

\section{Corresponding Author}

*Phone: +86-10-62923564; e-mail: zpinghao@rcees.ac.cn (Z.H.); phone: +61-8-8313-5657; e-mail: s.qiao@adelaide.edu. au (S.Q.).

Notes

The authors declare no competing financial interest.

\section{ACKNOWLEDGMENTS}

We gratefully acknowledge the financial support from the Natural Science Foundation of China (50978248, 20807050, 20725723), National Basic Research Program of China (2010CB732300), National High Technology Research and 
Development Program of China (2012AA063101), and the Australian Research Council (ARC) through Discovery Project program (DP0987969, DP1095861).

\section{REFERENCES}

(1) Ras, M. R.; Marce, R. M.; Borrull, F. Characterization of ozone precursor volatile organic compounds in urban atmospheres and around the petrochemical industry in the tarragona region. Sci. Total Environ. 2009, 407 (14), 4312-4319.

(2) Serrano, D. P.; Calleja, G.; Botas, J. A.; Gutierrez, F. J. Adsorption and hydrophobic properties of mesostructured MCM-41 and SBA-15 materials for volatile organic compound removal. Ind. Eng. Chem. Res. 2004, 43 (22), 7010-7018.

(3) Dou, B. J.; Li, J. J.; Hu, Q.; Ma, C. Y.; He, C.; Li, P.; Hu, Q. H.; Hao, Z. P.; Qiao, S. Z. Hydrophobic micro/mesoporous silica spheres assembled from zeolite precursors in acidic media for aromatics adsorption. Microporous Mesoporous Mater. 2010, 133 (1-3), 115123.

(4) Hu, Q.; Dou, B. J.; Tian, H.; Li, J. J.; Li, P.; Hao, Z. P. Mesoporous silicalite-1 nanospheres and their properties of adsorption and hydrophobicity. Microporous Mesoporous Mater. 2010, 129 (1-2), 30-36.

(5) Park, S. J.; Kim, Y. B.; Yeo, S. D. Vapor adsorption of volatile organic compounds using organically modified clay. Sep. Sci. Technol. 2008, 43 (5), 1174-1190.

(6) Qu, F.; Zhu, L. Z.; Yang, K. Adsorption behaviors of volatile organic compounds (VOCs) on porous clay heterostructures $(\mathrm{PCH})$. J. Hazard. Mater. 2009, 170 (1), 7-12.

(7) Hashisho, Z.; Emamipour, H.; Rood, M. J.; Hay, K. J.; Kim, B. J.; Thurston, D. Concomitant adsorption and desorption of organic vapor in dry and humid air streams using microwave and direct electrothermal swing adsorption. Environ. Sci. Technol. 2008, 42 (24), 9317-9322.

(8) Luo, L.; Ramirez, D.; Rood, M. J.; Grevillot, G.; Hay, K. J.; Thurston, D. L. Adsorption and electrothermal desorption of organic vapors using activated carbon adsorbents with novel morphologies. Carbon 2006, 44 (13), 2715-2723.

(9) Sullivan, P. D.; Rood, M. J.; Grevillot, G.; Wander, J. D.; Hay, K. $\mathrm{J}$. Activated carbon fiber cloth electrothermal swing adsorption system. Environ. Sci. Technol. 2004, 38 (18), 4865-4877.

(10) Emamipour, H.; Hashisho, Z.; Cevallos, D.; Rood, M. J.; Thurston, D. L.; Hay, K. J.; Kim, B. J.; Sullivan, P. D. Steady-state and dynamic desorption of organic vapor from activated carbon with electrothermal swing adsorption. Environ. Sci. Technol. 2007, 41 (14), 5063-5069.

(11) Sullivan, P. D.; Rood, M. J.; Dombrowski, K. D.; Hay, K. J. Capture of organic vapors using adsorption and electrothermal regeneration. J. Environ. Eng.-ASCE 2004, 130 (3), 258-267.

(12) Ramirez, D.; Emamipour, H.; Vidal, E. X.; Rood, M. J.; Hay, K. $\mathrm{J}$. Capture and recovery of methyl ethyl ketone with electrothermalswing adsorption systems. J. Environ. Eng.-ASCE 2011, 137 (9), 826832.

(13) Cui, X.; Shi, J.; Zhang, L.; Ruan, M.; Gao, J. PtCo supported on ordered mesoporous carbon as an electrode catalyst for methanol oxidation. Carbon 2009, 47 (1), 186-194.

(14) Qiao, W. M.; Song, Y.; Hong, S. H.; Lim, S. Y.; Yoon, S. H.; Korai, Y.; Mochida, I. Development of mesophase pitch derived mesoporous carbons through a commercially nanosized template. Langmuir 2006, 22 (8), 3791-3797.

(15) Yoon, S. B.; Chai, G. S.; Kang, S. K.; Yu, J. S.; Gierszal, K. P.; Jaroniec, M. Graphitized pitch-based carbons with ordered nanopores synthesized by using colloidal crystals as templates. J. Am. Chem. Soc. 2005, 127 (12), 4188-4189.

(16) Yang, Z. X.; Xia, Y. D.; Zhu, Y. Q.; Mokaya, R. Self-assembled ultralarge millimeter-sized graphitic carbon rods grown on mesoporous silica substrate. Chem. Mater. 2007, 19 (25), 6317-6322.
(17) Yang, C.; Weidenthaler, C.; Spliethoff, B.; Mayanna, M.; Schüth, F. Facile template synthesis of ordered mesoporous carbon with polypyrrole as carbon precursor. Chem. Mater. 2004, 17 (2), 355-358.

(18) Xia, Y. D.; Yang, Z. X.; Mokaya, R. Simultaneous control of morphology and porosity in nanoporous carbon: graphitic mesoporous carbon nanorods and nanotubules with tunable pore size. Chem. Mater. 2006, 18 (1), 140-148.

(19) Du, H.; Miller, J. D. Adsorption states of amphipatic solutes at the surface of naturally hydrophobic minerals: a molecular dynamics simulation study. Langmuir 2007, 23 (23), 11587-11596.

(20) Han, S. J.; Yun, Y. K.; Park, K. W.; Sung, Y. E.; Hyeon, T. Simple solid-phase synthesis of hollow graphitic nanoparticles and their application to direct methanol fuel cell electrodes. Adv. Mater. 2003, 15 (22), 1922.

(21) Wang, H. Y.; Abe, T.; Maruyama, S.; Iriyama, Y.; Ogumi, Z.; Yoshikawa, K. Graphitized carbon nanobeads with an onion texture as a lithium-ion battery negative electrode for high-rate use. Adv. Mater. 2005, 17 (23), 2857.

(22) González, D.; Montes-Morán, M. A.; Suárez-Ruiz, I.; Garcia, A. B. Structural characterization of graphite materials prepared from anthracites of different characteristics: a comparative analysis. Energy Fuels 2004, 18 (2), 365-370.

(23) Chai, S. H.; Howe, J. Y.; Wang, X. Q.; Kidder, M.; Schwartz, V.; Golden, M. L.; Overbury, S. H.; Dai, S.; Jiang, D. E. Graphitic mesoporous carbon as a support of promoted $\mathrm{Rh}$ catalysts for hydrogenation of carbon monoxide to ethanol. Carbon 2012, 50 (4), $1574-1582$

(24) Inagaki, M.; Kato, M.; Morishita, T.; Morita, K.; Mizuuchi, K. Direct preparation of mesoporous carbon from a coal tar pitch. Carbon 2007, 45 (5), 1121-1124.

(25) Morishita, T.; Ishihara, K.; Kato, M.; Inagaki, M. Preparation of a carbon with a $2 \mathrm{~nm}$ pore size and of a carbon with a bi-modal pore size distribution. Carbon 2007, 45 (1), 209-211.

(26) Shen, K.; Huang, Z. H.; Gan, L.; Kang, F. Y. Graphitic Porous Carbons Prepared by a Modified Template Method. Chem. Lett. 2009, 38 (1), 90-91.

(27) Li, J. J.; Mu, Z.; Xu, X. Y.; Tian, H.; Duan, M. H.; Li, L. D.; Hao, Z. P.; Qiao, S. Z.; Lu, G. Q. A new and generic preparation method of mesoporous clay composites containing dispersed metal oxide nanoparticles. Microporous Mesoporous Mater. 2008, 114 (1-3), 214-221.

(28) Yi, S. J.; Fan, Z.; Wu, C.; Chen, J. H. Catalytic graphitization of furan resin carbon by yttrium. Carbon 2008, 46 (2), 378-380.

(29) Sing, K. S. W.; Everett., D. H.; Haul, R. A. W.; Moscou, L.; Pierotti, R. A.; Rouquerol, J.; Siemieniewska, T. Reporting physisorption data for gas/solid systems with special reference to the determination of surface area and porosity. Pure Appl. Chem. 1985, 57 (4), 603-619. 